\title{
FGFR1 tyrosine kinase domain duplication in pilocytic astrocytoma with anaplasia
}

\author{
Leomar Y. Ballester, ${ }^{1}$ Marta Penas-Prado, ${ }^{2}$ Norman E. Leeds, ${ }^{3}$ Jason T. Huse, ${ }^{4}$ \\ and Gregory N. Fuller ${ }^{4}$ \\ ${ }^{1}$ Department of Pathology and Laboratory Medicine and Department of Neurosurgery, University of Texas \\ Health Science Center, Houston, Texas 77030, USA; ${ }^{2}$ Department of Neuro-Oncology, The University of Texas \\ MD Anderson Cancer Center, Houston, Texas 77030, USA; ${ }^{3}$ Department of Diagnostic Radiology, Section of \\ Neuroimaging, The University of Texas MD Anderson Cancer Center, Houston, Texas 77030, USA; \\ ${ }^{4}$ Department of Pathology, Section of Neuropathology, The University of Texas MD Anderson Cancer Center, \\ Houston, Texas 77030, USA
}

Abstract We report the case of a 27-yr-old male with visual field loss who had a 4.9-cm complex cystic mass in the right occipital lobe. Histologic examination showed pilocytic astrocytoma (PA) with anaplasia, and molecular characterization revealed FGFR1 duplication with additional variants of unknown significance in several genes (ARID1A, ARID1B, CHEK2, EPHA5, and MLL2). This is one of only a very few reported cases of anaplastic PA with characterization of molecular alterations.

Corresponding author: gfuller@mdanderson.org

(c) 2018 Ballester et al. This article is distributed under the terms of the Creative Commons Attribution License, which permits unrestricted reuse and redistribution provided that the original author and source are credited.

Ontology terms: astrocytoma

Published by Cold Spring Harbor Laboratory Press

doi: $10.1101 / \mathrm{mcs} . a 002378$

\section{CASE}

A 27-yr-old male presented to the emergency department with a complaint of left visual field loss after 2 mo of intermittent blurry vision. On examination, he had left homonymous hemianopsia. Magnetic resonance (MR) imaging demonstrated a $4.9 \times 2.7 \times 2.5$-cm complex multiloculated ring-enhancing lesion in the right occipital lobe with hemorrhagic foci (Fig. 1A). Right parieto-occipital lobe craniotomy with gross total resection of the tumor was performed.

Microscopic examination of the tissue showed a glial neoplasm with atypical cells displaying thin bipolar cytoplasmic processes (Fig. 1B), focally elevated mitotic activity (Fig. 1C), and prominent arcades of vascular proliferation that correlated with prominent ring enhancement seen on preoperative MR imaging (Fig. 1D). Tumor cell morphology varied from spindled to pleomorphic (Fig. 1E), with features of pilocytic astrocytoma (PA) and pleomorphic xanthoastrocytoma (PXA). Much of the tumor exhibited a prominent myxoid background (Fig. 1C,F); focal hyalinization of blood vessels was also observed. A relatively circumscribed interface with brain parenchyma was identified in several tissue fragments (Fig. 1D). Rosenthal fibers were not identified but EGBs were present (inset). Elevated mitotic activity was present (up to 7 mitoses per 10 high-power fields), and quantitative analysis yielded a correspondingly elevated Ki67 antigen (MIB1) labeling index (Fig. 1G).

Immunophenotyping showed focal areas of strong nuclear staining for p53 protein (Fig. $1 \mathrm{H})$. Molecular profiling of the tumor was performed using a next-generation sequencing assay that interrogates 315 genes for mutation, as well as the introns of 28 genes involved in gene rearrangements (FoundationOne assay, Foundation Medicine, INC). A genomic 

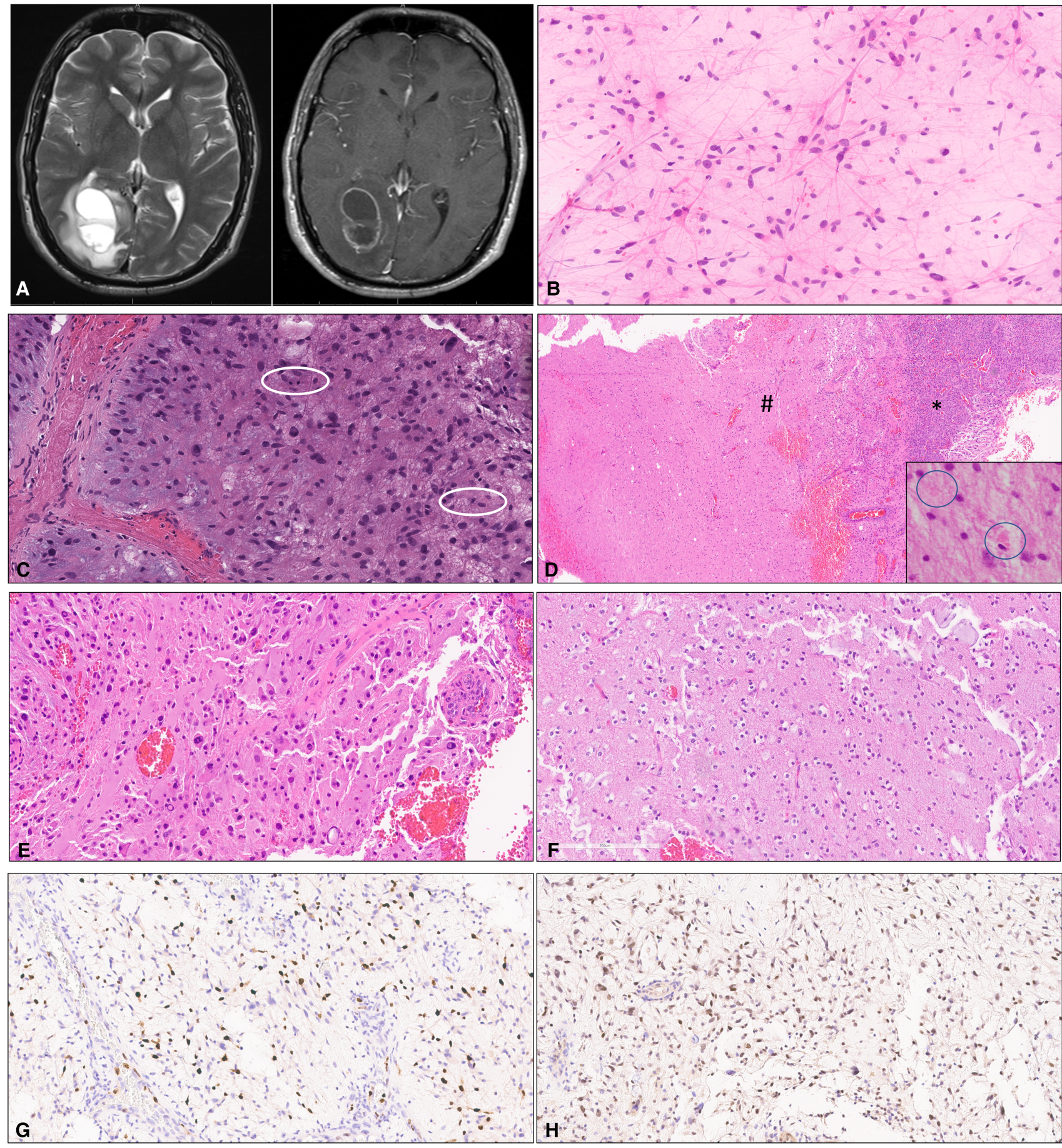

Figure 1. (A) Axial T2-weighted MRI sequence (left) and T1-weighted MRI sequence with contrast (right) showing the presence of a complex lesion in the right occipital lobe with ring enhancement. (B) Cytologic smear preparation showing neoplastic astrocytes with long, delicate bipolar cytoplasmic processes (pilocytic morphology). (C) Mitotic activity and myxoid background. (D) Arcade of vascular proliferation (*) and relatively sharp demarcation with adjacent brain parenchyma (\#). Rare eosinophilic granular bodies (EGBs) were present (inset). (E) Hypercellular brain parenchyma with pleomorphic tumor cells. (F) Tumor areas with myxoid appearance. (G) Ki67 antigen (MIB1) immunostain showing elevated labeling in the region with anaplasia. $(H)$ Expression of p53 protein by tumor cells in the region with anaplasia. 


\begin{tabular}{lllll}
\hline \multicolumn{4}{l}{ Table 1. Variants detected by next-generation sequencing analysis of tumor tissue } \\
\hline Gene & Chromosome & \multicolumn{1}{l}{ Position } & Variant type & Predicted effect \\
\hline FGFR1 & $8 \mathrm{p} 11.23$ & Exons 10-18 & Duplication & Pathogenic \\
ARID1A & $1 \mathrm{p} 36.11$ & p.P21_S22INSPP & Insertion/deletion & VUS \\
ARID1B & $6 \mathrm{q} 25.3$ & p.C251G & Missense mutation & VUS \\
CHEK2 & $22 \mathrm{q} 12.1$ & p.T367f*15 & Frameshift mutation & VUS \\
EPHA5 & $4 \mathrm{q} 13.2$ & p.D348G & Missense mutation & VUS \\
MLL2 & $12 q 13.12$ & p.E5292D & Missense mutation & VUS \\
\hline
\end{tabular}

VUS, variant of unknown significance.

alteration involving duplication of exons 10-18 of the FGFR1 gene was identified. In addition, five variants of unknown clinical significance were also identified (Table 1). Integration of the clinical, histologic, and molecular information available led to a final diagnosis of PA with anaplasia. Treatment with concurrent radiation and temozolomide was initiated.

\section{TECHNICAL ANALYSIS}

Molecular profiling of the tumor was performed using a next-generation sequencing assay that interrogates 315 genes for mutations, as well as the introns of 28 genes involved in gene rearrangements (FoundationOne assay, Foundation Medicine, INC). The test performs at a median depth of coverage of $500 \times$.

\section{DISCUSSION}

The diagnostic challenges associated with this case relate to the presence of histologic features that overlap between PXA and PA and the increased mitotic activity with vascular proliferation, focally elevated Ki67 index, and p53 staining in a young patient. PA occurs in adults, with lobar localization being a frequent finding ( $44 \%$ in some series) (Stüer et al. 2007). However, PA with anaplastic features at initial presentation is uncommon (Stüer et al. 2007; Azad et al. 2009). Anaplasia in PA has been associated with shortened survival (Rodriguez et al. 2010b). The current WHO 2016 Classification of Tumors of the Central Nervous System recognizes the existence of PA with anaplasia; however, specific grading criteria for this entity have yet to be defined (Louis et al. 2016).

Mitogen-activated protein kinase (MAPK) pathway alterations are common in PA; in fact, PA is considered to be predominantly a single pathway disease resulting from MAPK dysregulation (Jones et al. 2013; Pathak et al. 2017). PXA and PA were considered in the differential diagnosis of this case, although the classic genetic alterations present in PXA or PA (BRAF p.V600E mutation and BRAF-KIAA1549 fusion, respectively) were not present. However, an FGFR1 duplication, involving exons 10-18, which contains the protein tyrosine kinase domain (TKD), was identified. Duplication of the FGFR1 TKD has been reported in low-grade astrocytomas (including PA), usually in an extracerebellar location, and in dysembryoplastic neuroepithelial tumor (DNET) (Jones et al. 2013; Zhang et al. 2013; Rivera et al. 2016; Fina et al. 2017). In one study, 24\% of diffuse WHO 2007 grade II cerebral gliomas showed a duplication of the FGFR1 TKD. FGFR1 TKD duplication leads to autophosphorylation and activation of the MAPK/ERK and the PI3K pathways and has been shown to drive 
COLD SPRING HARBOR Molecular Case Studies
Anaplastic pilocytic astrocytoma with FGFR1 duplication
Competing Interest Statement

The authors have declared no competing interest.

\section{Referees}

Mariarita Santi

Kar-Ming Fung

Anonymous

Received November 15, 2017 accepted in revised form January 23, 2018. tumorigenesis (Zhang et al. 2013). The overall histologic features of this case are in favor of PA, and under the current classification system, this tumor is best classified as an anaplastic PA. Nonetheless, we recognize the possibility that the group of tumors with FGFR1 TKD duplication could represent a molecularly distinct subtype of glioma. However, further studies are required to confirm this assertion.

In addition to the FGFR1 TKD duplication, this case of PA with anaplasia showed additional genetic alterations of unknown biological and clinical significance (Table 1). The COSMIC, ExAC, and ClinVar databases were used to evaluate the potential significance of the additional variants detected in this case of PA with anaplasia. None of the variants is reported in the ExAC database. There is only a single entry of the ARID1A p21_S222INSPP variant in ClinVar (reported as likely benign), but we consider the evidence insufficient to make a final determination. There is one COSMIC entry for the CHEK2 T367fs*15 variant, and ClinVar shows 14 submissions of frameshift mutations in CHEK2, starting at codon 367. The variant is considered likely pathogenic in ClinVar. However, the significance of this variant in the context of our case is uncertain.

Genetic defects in ARID1A, ARID1B, and CHEK2 have been reported in brain tumors (Sallinen et al. 2005; Jones et al. 2012; Forbes et al. 2015; Park et al. 2015). It remains to be elucidated whether or not the FGFR1 duplication, or the additional genetic defects identified in this case, plays a direct role in the development of anaplastic features in PA. The molecular defects associated with PA have been characterized and primarily involve the MAPK pathway (e.g., BRAF-KIAA1549 fusion, BRAF p.V600E). However, the molecular defects associated with anaplasia in PA are not well known, although an association with neurofibromatosis type 1, the PI3K pathway, and p16 loss have been reported (Rodriguez et al. 2010a; Hsieh et al. 2012; Yeo et al. 2013). This case is one of only very few reports addressing the molecular defects involved in PA with anaplasia (Rodriguez et al. 2010a) and highlights the potential involvement of FGFR1 TKD in anaplastic PA.

\section{ADDITIONAL INFORMATION}

\section{Data Deposition and Access}

The variants were submitted to ClinVar (https://www.ncbi.nlm.nih.gov/clinvar/) and can be found under accession numbers SCV000692539, SCV000692540, SCV000692560, and SCV000692561.

\section{Ethics Statement}

The study was performed with approval of the Institutional Review Board (IRB) at the University of Texas MD Anderson Cancer Center (\# PA17-0216) with waiver of informed consent.

\section{REFERENCES}

Azad A, Deb S, Cher L. 2009. Primary anaplastic pilocytic astrocytoma. J Clin Neurosci 16: 1704-1706.

Fina F, Barets D, Colin C, Bouvier C, Padovani L, Nanni-Metellus I, Ouafik L, Scavarda D, Korshunov A, Jones DTW, et al. 2017. Droplet digital PCR is a powerful technique to demonstrate frequent FGFR1 duplication in dysembryoplastic neuroepithelial tumors. Oncotarget 8: 2104-2113.

Forbes SA, Beare D, Gunasekaran P, Leung K, Bindal N, Boutselakis H, Ding M, Bamford S, Cole C, Ward S, et al. 2015. COSMIC: exploring the world's knowledge of somatic mutations in human cancer. Nucleic Acids Res 43: D805-D811. 
COLD SPRING HARBOR Molecular Case Studies
Anaplastic pilocytic astrocytoma with FGFR1 duplication

Hsieh M-S, Ho JT-M, Lin L-W, Tu P-H, Perry A, Huang AP-H. 2012. Cerebellar anaplastic pilocytic astrocytoma in a patient of neurofibromatosis type-1: case report and review of the literature. Clin Neurol Neurosurg 114: 1027-1029.

Jones S, Li M, Parsons DW, Zhang X, Wesseling J, Kristel P, Schmidt MK, Markowitz S, Yan H, Bigner D, et al. 2012. Somatic mutations in the chromatin remodeling gene ARID1A occur in several tumor types. Hum Mutat 33: 100-103.

Jones DTW, Hutter B, Jäger N, Korshunov A, Kool M, Warnatz H-J, Zichner T, Lambert SR, Ryzhova M, Quang DAK, et al. 2013. Recurrent somatic alterations of FGFR1 and NTRK2 in pilocytic astrocytoma. Nat Genet 45: 927-932.

Louis DN, Ohgaki H, Wiestler OD, Cavenee WK, Ellison DW, Figarella-Branger D, Perry A, Reifenberger G, von Deimling A, eds. 2016. WHO classification of tumours of the central nervous system, 4th ed. WHO, Geneva.

Park C-K, Park I, Lee S, Sun C-H, Koh Y, Park S-H, Kim JE, Yun H, Lee S-H. 2015. Genomic dynamics associated with malignant transformation in IDH1 mutated gliomas. Oncotarget 6: 43653-43666.

Pathak P, Kumar A, Jha P, Purkait S, Faruq M, Suri A, Suri V, Sharma MC, Sarkar C. 2017. Genetic alterations related to BRAF-FGFR genes and dysregulated MAPK/ERK/mTOR signaling in adult pilocytic astrocytoma. Brain Pathol 27: 580-589.

Rivera B, Gayden T, Carrot-Zhang J, Nadaf J, Boshari T, Faury D, Zeinieh M, Blanc R, Burk DL, Fahiminiya S, et al. 2016. Germline and somatic FGFR1 abnormalities in dysembryoplastic neuroepithelial tumors. Acta Neuropathol 131: 847-863.

Rodriguez EF, Scheithauer BW, Giannini C, Rynearson A, Cen L, Hoesley B, Gilmer-Flynn H, Sarkaria JN, Jenkins S, Long J, et al. 2010a. PI3K/AKT pathway alterations are associated with clinically aggressive and histologically anaplastic subsets of pilocytic astrocytoma. Acta Neuropathol 121: 407-420.

Rodriguez FJ, Scheithauer BW, Burger PC, Jenkins S, Giannini C. 2010b. Anaplasia in pilocytic astrocytoma predicts aggressive behavior. Am J Surg Pathol 34: 147-160.

Sallinen S-L, Ikonen T, Haapasalo H, Schleutker J. 2005. CHEK2 mutations in primary glioblastomas. J Neurooncol 74: 93-95.

Stüer C, Vilz B, Majores M, Becker A, Schramm J, Simon M. 2007. Frequent recurrence and progression in pilocytic astrocytoma in adults. Cancer 110: 2799-2808.

Yeo YH, Byrne NP, Counelis GJ, Perry A. 2013. Adult with cerebellar anaplastic pilocytic astrocytoma associated with BRAF V600E mutation and p16 loss. Clin Neuropathol 32: 159-164.

Zhang J, Wu G, Miller CP, Tatevossian RG, Dalton JD, Tang B, Orisme W, Punchihewa C, Parker M, Qaddoumi I, et al. 2013. Whole-genome sequencing identifies genetic alterations in pediatric low-grade gliomas. Nat Genet 45: 602-612. 


\section{COLD SPRING HARBOR Molecular Case Studies}

\section{FGFR1 tyrosine kinase domain duplication in pilocytic astrocytoma with anaplasia}

Leomar Y. Ballester, Marta Penas-Prado, Norman E. Leeds, et al.

Cold Spring Harb Mol Case Stud 2018, 4: a002378

Access the most recent version at doi: $10.1101 /$ mcs.a002378

License This article is distributed under the terms of the Creative Commons Attribution License, which permits unrestricted reuse and redistribution provided that the original author and source are credited.

Email Alerting Receive free email alerts when new articles cite this article - sign up in the box at the Service top right corner of the article or click here. 